\title{
Individual differences in conflict-monitoring: testing means and covariance hypothesis about the Simon and the Eriksen Flanker task
}

\author{
Doris Keye · Oliver Wilhelm • Klaus Oberauer · \\ Don van Ravenzwaaij
}

Published online: 1 September 2009

(C) Springer-Verlag 2009

\section{Erratum to: Psychol Res \\ DOI 10.1007/s00426-008-0188-9}

The authors regret that due to an error in the stimulus coding, the discrimination of stimulus repetitions from stimulus alternations was not correct in the published data. The error was corrected and reanalyses were performed. The following changes emerged in these corrections:

We find main effects of stimulus repetition in reaction time (RT) as well as accuracies in ANOVAs for the Eriksen and the Simon task. Regarding the Eriksen task, the main effect of stimulus repetition is relatively small (RT: partial $\eta^{2}$ 0.03; Accuracy: partial $\eta^{2} 0.28$ ), but an interaction effect with previous trial compatibility (RT: partial $\eta^{2} 0.31$; Accuracy: partial $\eta^{2}$ 0.59) indicates that repetition effects are present, especially in iI (incompatible trials that are preceded by incompatible trials) trials and also $\mathrm{cC}$ trials. We

The online version of the original article can be found under doi:10.1007/s00426-008-0188-9.

Electronic supplementary material The online version of this article (doi:10.1007/s00426-009-0257-8) contains supplementary material, which is available to authorized users.

D. Keye $(\varangle) \cdot$ O. Wilhelm

Institute for Progress in Education, Humboldt-University Berlin,

Unter den Linden 6, 10099 Berlin, Germany

e-mail: doris.keye@iqb.hu-berlin.de

K. Oberauer

Department of Experimental Psychology,

University of Bristol, Bristol, UK

D. van Ravenzwaaij

Department of Psychological Methods,

University of Amsterdam, Amsterdam, The Netherlands find stronger repetition effects for the Simon task data (RT: partial $\eta^{2}$ 0.45; Accuracy: partial $\eta^{2}$ 0.39), but interactions with previous trial compatibility are smaller (RT: partial $\eta^{2}$ 0.22; Accuracy: partial $\eta^{2}$ 0.12). Again, the repetition effect is strongest for the iI Simon trials. Effects of previous trial compatibility remain after controlling for repetition priming (Eriksen task: RT: partial $\eta^{2}$ 0.11; Accuracy: partial $\eta^{2}$ 0.61; Simon task: RT: partial $\eta^{2} 0.36$; Accuracy: partial $\eta^{2}$ $0.67)$.

We reran all structural equation analyses. Regarding RT data, the introduction of a repetition factor loading on all indicators that are based on identical stimulus successions was necessary for both tasks to arrive at acceptable measurement models (Eriksen task: $\chi^{2}=33, \mathrm{df}=10, p<0.01$, CFI $=0.99$, SRMR $=0.01$; Simon task: $\chi^{2}=31, \mathrm{df}=8$, $p<0.01, \mathrm{CFI}=0.99$, SRMR $=0.01$ ). The variance of the context factor decreased after introducing the repetition factor as such that it was no longer necessary to keep the factor. Correlations among nested factors were not observed for the Eriksen task. Concerning the Simon task, the conflict and the context factor still correlated significantly at $0.41\left(\chi^{2}=26, \mathrm{df}=7, p<0.01, \mathrm{CFI}=0.99\right.$, SRMR $=0.01)$. A constrained measurement model combining RT indicators of both tasks fitted the data still satisfactory $\left(\chi^{2}=366, \mathrm{df}=90, p<0.01, \mathrm{CFI}=0.94\right.$, SRMR $\left.=0.08\right)$. Associations between experimental factors and between experimental factors and the criteria working memory (WM) and factors of impulsivity did not change relative to the published data.

For the accuracy data the measurement model for the Eriksen task incorporated a general performance factor and nested factors for conflict and repetition priming $\left(\chi^{2}=10\right.$, $\mathrm{df}=12, p=0.59, \mathrm{CFI}=1.00, \mathrm{SRMR}=0.02)$. Constraining loadings deteriorated fit significantly $\left(\Delta \chi^{2}=78, \mathrm{df}=11\right)$. The measurement model for the Simon accuracy data with 
one general performance factor and nested factors for conflict, context, and repetition priming fitted very well $\left(\chi^{2}=5\right.$, $\mathrm{df}=6, p=0.73, \mathrm{CFI}=1.00$, SRMR $=0.01$ ). Constraining the loadings again did not result in an acceptable model $\left(\Delta \chi^{2}=49, \mathrm{df}=15\right)$. A combined model for both tasks fitted the data $\left(\chi^{2}=76, \quad \mathrm{df}=70, \quad p=0.30, \quad \mathrm{CFI}=0.99\right.$, SRMR $=0.03$ ), and correlations did not differ from those originally reported. The repetition factors from both tasks correlated with each other at $0.46\left(\Delta \chi^{2}=17, \mathrm{df}=1\right)$, but showed no associations with other factors, including WM and impulsivity factors, and importantly did not affect correlations of other Simon and Eriksen factors with criteria.

Concluding, deviating from the initial analysis we find repetition effects for mean RT and mean accuracies as well as individual differences in these mean effects. Repetition priming does not account for the context slow-down effects completely, though weakening the initially reported effects. The task-specific repetition factors were correlated but far from forming a coherent repetition factor. The repetition factors have no consistent substantial correlations with WM and self-reported impulsivity. The introduction of the repetition factors leaves associations with other experimental factors and criteria essentially unaffected, and therefore none of the conclusions from the published paper-apart from those concerning the repetition effect-needs adjustment or modification. An exhausting depiction of the results is available online as electronic supplementary material. 